\title{
O autocuidado de homens e mulheres com Diabetes Mellitus tipo 2
}

\author{
The men and woman's selfcare with type 2 Diabetes Mellitus \\ Autocuidado de hombres y mujeres con Diabetes Mellitus tipo 2
}

Recebido: 04/01/2021 | Revisado: 08/01/2021 | Aceito: 11/01/2021 | Publicado: 13/01/2021

Letiane de Oliveira Rubira

ORCID: https://orcid.org/0000-0003-2443-608X Universidade Federal do Rio Grande, Brasil E-mail: letianerubira@gmail.com

Victoria Leslyê Rocha Gutmann

ORCID: https://orcid.org/0000-0002-3457-7620 Universidade Federal do Rio Grande, Brasil E-mail: victorialeslye@gmail.com

Vanessa Machado da Silva

ORCID: https://orcid.org/0000-0002-4319-4509 Universidade Federal do Rio Grande, Brasil E-mail: va.nessamachado@outlook.com

Marina Soares Mota

ORCID: https://orcid.org/0000-0002-5717-9406 Universidade Federal de Pelotas, Brasil

E-mail:msm.mari.gro@gmail.com

Fabiane Ferreira Francioni

ORCID: https://orcid.org/0000-0002-3384-0802 Universidade Federal do Rio Grande, Brasil E-mail: francionifabiane@gmail.com

Diéssica Roggia Piexak

ORCID: https://orcid.org/0000-0002-3374-7843 Universidade Federal do Rio Grande, Brasil E-mail: diessicap@yahoo.com.br

Camila Daiane Silva

ORCID: https://orcid.org/0000-0002-0739-4984

Universidade Federal do Rio Grande, Brasil

E-mail: camilad.silva@yahoo.com.br

\begin{abstract}
Resumo
Objetivo: conhecer o autocuidado de homens e mulheres com Diabetes Mellitus tipo 2. Metodologia: estudo qualitativo, descritivo. Dados coletados entre julho e agosto de 2019 por meio de entrevista semiestruturada. Participaram 14 pessoas, sendo sete homens e sete mulheres com Diabetes Melittus tipo 2 atendidos no Centro Integrado de Diabetes de um Hospital Universitário. Tratamento dos dados por meio da Análise de Conteúdo proposta por Bardin. Resultados: a análise resultou em quatro categorias, denominadas "Conhecimento sobre o que é autocuidado frente ao DM2 e a participação familiar"; "Aspectos gerais do autocuidado praticado acerca do DM2"; "Comportamento alimentar versus o autocuidado"; e "A rotina de vida versus a realização de atividade física". Comportamentos similares de autocuidado foram encontrados entre homens e mulheres, tais como o cuidado com a alimentação, com os pés e com a medicação, além da realização de exercícios físicos. Neste último, verificou-se que as mulheres estão mais envolvidas nas tarefas do lar e cuidados com outros membros de sua família, o que as distancia, quando comparado aos homens, das práticas de atividades físicas, evidenciando diferenças entre os gêneros. Considerações finais: para melhorar o autocuidado das pessoas com diabetes, faz-se necessário um maior engajamento e conscientização dos mesmos sobre sua saúde com mudanças no estilo de vida. Esse processo pode ser facilitado por meio de intervenções dos profissionais da saúde, em especial da enfermagem, no que diz respeito à educação em saúde dos indivíduos, levando em consideração as questões de gênero existentes que podem influenciar o autocuidado.
\end{abstract}

Palavras-chave: Diabetes Mellitus Tipo 2; Autocuidado; Gênero e saúde; Enfermagem.

\begin{abstract}
Objective: know the self-care of men and women with Diabetes Mellitus type 2. Methodology: Qualitative and descriptive study. Data were collected between July 2019 to august 2019 by semi-structured interviews. 14 people have participate, seven of them were men and seven women with diabetes type 2 treated in the Integrated Center of diabetes on a University Hospital. Results: The analysis found 4 categories named "Knowledge about what is selfcare face to DM2 e family participation"; "General aspects of self-care about DM2"; "Eat behavior versus self-care"," The Life routine versus the physical activity". Similarities behaviors were found between men and women, as such as care with diet, with feet and medication, beyond performing physical activities. In the last year, was checked that
\end{abstract}


women are more involved in house tasks and take care of family members, that compared to men, reduce the practice of physical activities, highlighting the disparities between genders. Final considerations: To improve the people selfcare with diabetes, is necessary a bigger commitment and consciousness of themselves about their health with life chance style. This process can be facilitated by health professional interventions, in special of nursing, concerning to individual health, taking on consideration gender questions relevant that could affect the self-care.

Keywords: Diabetes Mellitus Type 2; Self care; Gender and health; Nursing.

\begin{abstract}
Resumen
Objetivo: conocer el autocuidado de hombres y mujeres con Diabetes Mellitus tipo 2 Metodología: estudio descriptivo cualitativo. Datos recolectados entre julio y agosto de 2019 mediante entrevistas semiestructuradas. Participaron 14 personas, siete hombres y siete mujeres con Diabetes Melittus Tipo 2 tratados en el Centro Integrado de Diabetes de un Hospital Universitario. Tratamiento de datos mediante análisis de contenido propuesto por Bardin Resultados: el análisis resultó en cuatro categorías, denominadas "Conocimiento sobre qué es el autocuidado frente a la DM2 y la participación familiar"; "Aspectos generales del autocuidado practicado sobre la DM2"; "Comportamiento alimenticio versus autocuidado"; y "La rutina de la vida versus la actividad física". Se encontraron comportamientos similares de autocuidado entre hombres y mujeres, como cuidar la comida, los pies y la medicación, además del ejercicio físico. En este último, se encontró que las mujeres se involucran más en las tareas del hogar y el cuidado de otros miembros de su familia, lo que las aleja, en comparación con los hombres, de las prácticas de actividad física, mostrando diferencias entre géneros. Consideraciones finales: Para mejorar el autocuidado de las personas con diabetes, es necesario un mayor compromiso y conciencia sobre su salud con los cambios en el estilo de vida. Este proceso puede ser facilitado mediante intervenciones de los profesionales de la salud, especialmente de enfermería, con respecto a la educación para la salud de las personas, teniendo en cuenta las cuestiones de género existentes que pueden influir en el autocuidado.
\end{abstract}

Palabras clave: Diabetes Mellitus Tipo 2; Autocuidado; Género y salud; Enfermería.

\title{
1. Introdução
}

As Doenças Crônicas Não Transmissíveis (DCNT) são definidas como doenças de múltiplas causas e fatores de risco, como uso de tabaco, consumo excessivo de álcool, hábitos alimentares pouco saudáveis e a não realização de atividade física, caracterizadas por um curso prolongado, de início não infeccioso, e também por estarem associadas a incapacidades funcionais e deficiências (World Health Organization, 2014). Dentre as diversas doenças definidas como DCNT, destacam-se as cardiovasculares, as neoplasias, as doenças respiratórias crônicas e o Diabetes Mellitus (DM) (Brasil, 2018).

Em relação ao DM, seu conceito é definido pela Sociedade Brasileira de Diabetes (SBD) como um distúrbio metabólico resultante da deficiência na produção de insulina e/ou de sua ação, levando à hiperglicemia persistente e ocasionando complicações a longo prazo (Sociedade Brasileira de Diabetes, 2017). O DM é classificado em diversos tipos, como por exemplo, o tipo 1, o tipo 2, o gestacional, entre outros (International Diabetes Federation, 2017).

O DM tipo 2 (DM2), sendo o foco central deste trabalho e o mais frequente, apresenta caráter genético ainda pouco esclarecido, além dos fatores ambientais que contribuem para o seu desenvolvimento. Essa patologia leva à perda progressiva na produção de insulina aliada a resistência insulínica periférica (Sociedade Brasileira de Diabetes, 2017). O DM2 é encontrado com maior frequência em adultos com idade mais avançada, contudo tem acometido crianças, adolescentes e adultos jovens em virtude dos estilos de vida que favorecem a obesidade (International Diabetes Federation, 2017).

O controle da doença através de mudanças no estilo de vida, adesão às ações de promoção e manutenção à saúde são objetivos dos profissionais de saúde, de modo a incentivar que os pacientes assumam comportamentos saudáveis. O enfermeiro atua como facilitador e exerce grande influência no estímulo do paciente para realizar seu plano de autocuidado, levando em conta os fatores de risco apresentados durante sua assistência. Dessa forma, também compete ao enfermeiro incentivar a prática de exercícios físicos regulares e uma alimentação equilibrada, contribuindo para o bom controle glicêmico (Araújo, et al., 2018), além da redução do consumo de bebidas alcoólicas e cessação do tabagismo (Brasil, 2013).

Entretanto, sabe-se que as diferenças socioeconômicas e fatores como idade, nível de escolaridade e questões culturais relacionadas ao gênero podem influenciar as percepções sobre saúde e a busca pelos serviços de saúde entre homens e mulheres (Moura, Gomes, \& Pereira, 2017). O conceito de gênero está relacionado aos aspectos subjetivos e culturais impostos 
e reproduzidos pela sociedade ao longo dos anos do que é ser mulher ou ser homem (Oka, \& Laurenti, 2018). Logo, a educação para o autocuidado deve ser realizada levando-se em conta a singularidade das pessoas, uma vez que as questões de gênero podem afetar diretamente o estilo de vida das mesmas, bem como a aceitação da terapêutica e adesão ao tratamento de uma doença crônica como o DM2.

Desse modo é necessário entender o que os indivíduos compreendem por autocuidado e como o desempenham em sua rotina diária, bem como se existe ausência ou limitação do exercício em virtude das questões de gênero. Assim, essa pesquisa tem como questão norteadora: como se desenvolve o autocuidado de homens e mulheres com Diabetes Mellitus tipo 2 ? Portanto, objetivou-se conhecer o autocuidado de homens e mulheres com Diabetes Mellitus tipo 2.

\section{Metodologia}

Trata-se de um estudo descritivo, com abordagem qualitativa. A pesquisa descritiva se apoia principalmente em estudos qualitativos e têm como objetivo apresentar resumos do senso comum (Polit, \& Beck, 2011). Participaram da pesquisa 14 pessoas com diagnóstico de Diabetes Mellitus tipo 2, sendo sete homens e sete mulheres, em acompanhamento no Centro Integrado de Diabetes (CID) do Hospital Universitário Dr. Miguel Riet Corrêa Jr. da Universidade Federal do Rio Grande (HU-FURG), vinculado à Empresa Brasileira de Serviços Hospitalares (EBSERH).

Os participantes foram convidados conforme a livre demanda do serviço, até se atingir a saturação dos dados. A saturação indica o ponto em que a inclusão de informações se torna desnecessária, porque não modifica o entendimento do evento estudado (Thiry-Cherques, 2009). Os critérios de inclusão foram homens e mulheres com DM2, atendidos no CID, com idade superior a 18 anos, que tivessem feito mais de uma consulta ambulatorial. Enquanto que os critérios de exclusão foram usuários com DM1, DMG, outras patologias que não fossem DM2 e menores de idade.

No primeiro contato, ocorreu o processo de consentimento, sendo orientado verbal e individualmente cada participante, entregando o Termo de Consentimento Livre e Esclarecido para expressão do aceite através da assinatura em duas vias, uma para ele e outra para o pesquisador. Para a coleta dos dados, que ocorreu no período entre julho e agosto de 2019, foi elaborado um roteiro de entrevista, com perguntas abertas referentes ao conhecimento acerca do diabetes, da percepção de seu autocuidado e da adesão ao tratamento, além de perguntas fechadas sobre os aspectos socioeconômicos para caracterizar os participantes.

As entrevistas, gravadas com o uso de gravador digital e transcritas posteriormente, ocorreram em sala reservada no HU-FURG, localizada próxima ao CID, longe de ruídos externos e interrupções, sem interferir o andamento do serviço. Os dados foram analisados por meio da Análise de Conteúdo proposta por Bardin, estruturada em três momentos - pré-análise; exploração do material; e tratamento dos dados obtidos, inferência e interpretação. A pré-análise é a fase da organização e sistematização das ideias introdutórias, selecionando os materiais que serão submetidos à análise e formando hipóteses e objetivos. A exploração do material é considerada uma fase extensa que compreende a codificação, a decomposição ou a enumeração dos dados não tratados. $\mathrm{O}$ tratamento dos resultados obtidos, a inferência e a interpretação, baseiam-se em evidenciar os dados significativos subordinando-os a testes estatísticos e de validação. Desse modo, é possível sugerir inferências e interpretações dos objetivos esperados (Bardin, 2011).

Destaca-se que a coleta dos dados iniciou somente após a aprovação do Comitê de Ética, de modo que a pesquisa obteve parecer $\mathrm{n}^{\circ}$ 88/2019 e CAEE 16541519.5.0000. Para a garantia do anonimato, os participantes foram identificados pela letra "F" se sexo feminino e "M" se sexo masculino, seguido do número da ordem de realização das entrevistas.

\section{Resultados e Discussão}

Dos 14 participantes, sete eram homens e sete eram mulheres. As idades variaram entre 56 e 76 anos. Com relação ao 
estado civil, 50\% tinham companheiro e moravam com ele, $42,9 \%$ não tinham companheiro e moravam sozinhos e $7,1 \%$ tinham companheiro e não moravam com ele. No que se refere à escolaridade, $85,7 \%$ tinham ensino fundamental completo/incompleto e 14,3\% referiram ter ensino médio completo/incompleto. Com relação ao DM2, o tempo de diagnóstico variou entre 4 a 28 anos. O tempo de tratamento variou entre 4 e 20 anos. Os anos de acompanhamento no CID variaram entre 1 e 20 anos. A maior parte dos participantes, 64,3\%, disseram não realizar acompanhamento na unidade básica de saúde, enquanto que 35,7\% afirmaram fazer esse acompanhamento já por um tempo variado entre um e oito anos. Somente 14,3\% disseram participar de algum grupo de apoio à pessoa com diabetes e 85,7\% não faziam parte de nenhum grupo.

A análise dos dados resultou em quatro categorias, respectivamente denominadas: "Conhecimento sobre o que é autocuidado frente ao DM2 e a participação familiar"; "Aspectos gerais do autocuidado praticado acerca do DM2"; "Comportamento alimentar versus o autocuidado"; e "A rotina de vida versus a realização de atividade física".

\section{Conhecimento sobre o que é autocuidado frente ao DM2 e a participação familiar}

Com relação ao conhecimento dos participantes sobre o que é autocuidado, verificou-se incertezas nas respostas de homens e mulheres. Mesmo quando havia um pouco mais de propriedade do assunto, o conhecimento ainda era expresso com fragilidade. Identificou-se, ainda, o desejo dos participantes em melhorar o seu autocuidado, pois reconheceram existir falhas.

Autocuidado é a pessoa se cuidar?! (M11)

É não fazer as coisas erradas... Eu acho. (F6)

Acho que o autocuidado é tudo. É dedicação em primeiro lugar, estar certinha com a medicação e a alimentação. (F9)

Autocuidado é se cuidar, entendeu?! Não fazer coisa errada. Não comer as coisas que não pode comer, não abusar. Ter calma, não ter ansiedade, como às vezes eu tenho. (M5)

Autocuidado? É cuidar da alimentação, cuidar da higiene, dos pés... É tomar consciência que preciso me cuidar. (F3) Pode melhorar, com certeza. (F9)

É deficiente. Deveria melhorar. (M11)

O autocuidado é indispensável na prevenção e adiamento de consequências negativas advindas do diabetes, além de permitir um declínio nos índices de internações hospitalares decorrentes da patologia (Hoerpers, Roldão, Fernandes, Dimer, \& Pavei, 2019). Uma pesquisa realizada no Ceará em 2016, demonstrou que a conscientização a respeito dos cuidados necessários no controle do diabetes é bastante relevante, levando em consideração as boas práticas de saúde, bem como a realização de atividades físicas, adesão ao tratamento medicamentoso, bons hábitos alimentares e o cuidado e a higiene com os pés (Trajano, et al., 2018).

Identificou-se que os participantes, tanto homens quanto mulheres, acreditavam realizar um bom e suficiente autocuidado. Porém, especialmente na questão alimentar, referiram ingerir carboidratos em excesso, evidenciando uma visão equivocada do que é um autocuidado ideal em relação ao DM2.

Acho que é bom. Tem que se cuidar. Acho que eu me cuido, porque sei que depois é pior. (M 10)

Eu me cuido. Tem que ter saco [paciência] para cuidar do glaucoma, tomar remédio para pressão e ainda cuidar do 
diabetes... Tem que ter disciplina. Se não tiver, não dá, já era. (M 12)

[...] Tem dias que almoço e tem dias que não almoço, faço um lanche, às vezes pão, bolachinha, uma recheada, e às vezes uma pizza no final de semana. (F3)

A construção de um planejamento de cuidados a partir das necessidades de cada paciente deve ser realizada em conjunto com o enfermeiro e a equipe de saúde, de modo a gerar reflexão sobre o autocuidado, considerando as vulnerabilidades e particularidades desses indivíduos. Dessa forma, poderá ser prestada uma melhor assistência, visando o esclarecimento sobre as dificuldades enfrentadas e desenvolvendo uma conduta que seja efetiva no cuidado das pessoas com DM2 (Costa, et al., 2016).

Quanto ao envolvimento familiar, percebeu-se que a família não incentiva o autocuidado por residir longe, por individualidade ou até mesmo por descaso.

Não participam, não. Ninguém fala nada. Eu moro só e a família longe. (M10)

Não participa. (F2)

Ninguém participa de nada. Cada um sabe de si! (M12)

Ah, eles moram tudo longe de mim, eles nem sabem. (F9)

Por outro lado, uma pesquisa identificou que os familiares se preocupam com os parentes acometidos pela doença, se importam com o cuidado da terapêutica prescrita pelo profissional de saúde, ocorrendo uma mudança nos hábitos de toda a família. Ademais, a família é encarada como rede de apoio no cuidado dos indivíduos com doenças crônicas. Logo, a equipe de saúde pode abordar os cuidados também com os familiares, a fim de incentivá-los a realizar modificações necessárias no estilo de vida e nas adaptações pertinentes no processo da patologia e na adesão ao tratamento do paciente (Martins, \& Rodrigues, 2019). Apesar da família não contribuir no estímulo para o autocuidado, os participantes referiram que possuíam pelo menos um familiar de primeiro grau também acometido pelo DM2.

Tem. Minha mãe. Só minha mãe. (M5)

A minha irmã. (F9)

Tem. A minha mãe, o meu filho e meus irmãos, três irmãos. (F7)

Para a Sociedade Brasileira de Diabetes, a história familiar da doença é um fator de risco, principalmente se em familiares de primeiro grau e associada a outros fatores de risco. Uma pesquisa realizada em Sergipe, em 2018, destacou que as pessoas com quadro de hiperglicemia prévia associada ao histórico familiar de algum membro da família de primeiro grau com DM apresentaram um risco alto ou muito alto de desenvolver a doença (Lisley, 2019).

\section{Aspectos gerais do autocuidado praticado acerca do DM2}

No que se refere aos principais autocuidados que uma pessoa com DM2 deve seguir para manutenção de sua saúde, foram elencadas de imediato a alimentação e a atividade física. 
Exercício físico, cuidar da alimentação, ter consciência dos perigos que envolvem. (F3)

Todos. Alimentação, fazer exercício... (F7)

Seguinte: o importante é fazer exercício, se movimentar. Não pode ficar sedentário. É isso [risos]. Não comer doce [risos]. Fazer uma dieta mais ou menos. (M13)

Das práticas de autocuidado elencadas, questionou-se quais eram praticadas em sua rotina de vida. Verificou-se que a alimentação e a realização de exercício físico foram prontamente lembradas, além disso, o uso de medicações e o cuidado com os pés.

[...] Controlo a alimentação, o exercício, não comer o que não posso comer, no caso pão, arroz, massa... (M13)

Eu me cuido. Alimentação, faço atividade física, exercício. (M5)

Evito comer coisas doces, muita massa e coisas assim, além de cuidar da medicação. (M11)

A gente tem que ter cuidado em tudo. Tanto nos pés, como nas mãos, no corpo. Tem que estar sempre hidratando e tomar a medicação. (F9)

Um estudo realizado no norte do Paraná com 18 pessoas com DM2 revelou que elas identificam e aceitam os comportamentos necessários frente à doença, como a disciplina alimentar, a realização de atividade física e o controle do nervosismo. Apesar disso, não realizam ou não dão continuidade a esses comportamentos por escolha individual, por causas externas, como o arranjo do sistema de saúde que não fornece orientação, ou a faz de forma sucinta, além dos embates com a família (Teston, Sales, \& Marcon, 2017).

O autocuidado com os pés foi considerado muito importante para quem convive com o DM2. Verificou-se o sentimento de preocupação entre os participantes, os quais referiram ter um bom cuidado com seus pés.

Eu cuido. Tem um creme que estou passando nos pés, também cuido para não cortar os pés, não machucar. (F2)

Cuido. De vez em quando olho meu pé. Quando tomo banho olho e seco bem sequinho no meio dos dedos. Às vezes está ressecado e passo óleo. (M5)

\section{[...] Cuido da unha. Não machuco o pé. (M12)}

Uma pesquisa que incluiu os resultados de estudos realizados entre 2010 e 2017, apontou que as pessoas com DM demonstraram erros nas ações que previnem o pé diabético, o que pode acarretar em um aumento das chances de desenvolver complicações e limitações com danos à saúde. Há um déficit de conhecimento de atitudes que previnam maiores problemas nos pés de pessoas com DM. Os erros cometidos por eles foram relacionados ao conhecimento deficiente por falta de informações a respeito da higiene, inspeção, secagem e hidratação dos pés, além de não saberem o corte correto das unhas, qual melhor calçado para usar, qualidade das meias, e intervenções que se não realizadas podem trazer prejuízos à saúde dos indivíduos. Com isso, compete aos profissionais de saúde, em especial o enfermeiro, orientar quanto aos cuidados com os pés (Ribeiro, \& Nunes, 2018).

Nessa vertente, outra pesquisa evidenciou que o enfermeiro exerce grande influência na assistência à pessoa com 
DM2. Esse profissional pode atuar no que diz respeito ao atendimento relacionado ao diagnóstico, prevenção e tratamento da comorbidade em questão, uma vez que está intimamente relacionada com a diminuição da qualidade de vida dos pacientes e com os níveis de mortalidade (Pimentel, \& Marques, 2019).

Outro autocuidado elencado foi o uso correto da medicação para o DM2. Os participantes demonstraram uma boa adesão ao tratamento farmacológico, não somente em relação ao DM2, como também ao uso de medicações para suas comorbidades.

[...] Insulina e o Glifage. Tomo a insulina 30 e dez. 30 de manhã e dez à noite. E dois Glifage antes da janta ou pósjanta, mas é antes da janta. (M5)

Tomo Glifage, dois no almoço e dois na janta. E a insulina, 14 de manhã e 16 à noite. (F2)

É o Glifage e o Losartana. Agora ela colocou esse aqui [glicasida]. O glifage são dois de manhã e dois à noite e agora quatro comprimidos daquele [glicasida] à noite, um comprimido dez minutos antes do almoço [lido na receita]. (M14)

Independentemente do sexo, homens e mulheres, relataram o uso de medicamento oral e insulina, indicando, possivelmente, que a taxa glicêmica é tão alta que a insulina endógena associada aos medicamentos orais não são o suficiente para eles. Ao encontro deste estudo, uma pesquisa realizada em Brasília com pessoas com DM2 evidenciou que grande parte dos entrevistados faziam uso de metformina e apresentavam comorbidades associadas ao diabetes, como hipertensão e obesidade grau I. A dislipidemia foi encontrada em menos da metade da amostra em virtude do uso de estatinas (Cruz, et al., 2019).

Um estudo revelou que os participantes apresentaram melhor adesão do tratamento farmacológico em comparação ao não farmacológico, porque este último exige autodomínio, encorajamento e mudanças no estilo de vida. Os indivíduos demonstraram disciplina no uso correto das medicações, contribuindo para a obtenção de bons resultados no tratamento de um modo geral (Silva, \& Alves, 2018). Da mesma forma, outra pesquisa com o objetivo de analisar o conhecimento de pessoas diabéticas e sua associação com a adesão ao autocuidado e controle glicêmico constatou a boa adesão ao tratamento medicamentoso e a monitorização da glicemia em comparação a outros cuidados, tais como o cuidado com os pés, a alimentação geral e a atividade física, que obtiveram baixa adesão (Silva, et al., 2020).

Por outro lado, outra pesquisa realizada em idosos com DM2 na Bahia, no ano de 2018, identificou a baixa adesão, não proposital, ao tratamento medicamentoso. Isso foi causado pelo esquecimento e atraso nos horários das medicações para o DM2. Essas atitudes frente ao uso das medicações pode ser consequência das alterações cognitivas da idade (Fernandes, Damascena, \& Portela, 2019).

A realização do hemoglicoteste foi outro autocuidado elencado, sendo este o de maior adesão entre os participantes. No entanto, realizam apenas uma vez por semana pela falta do aparelho em casa, necessitando o deslocamento até uma unidade de saúde para realização do teste.

Sim, todos os dias. (F9)

Às vezes quando dá tempo, uma vez por semana. (M14)

Realizo, toda quarta-feira, porque é no posto. Eu não tenho aparelho então toda quarta feira vou lá. (M13)

Ao encontro deste resultado, uma pesquisa realizada com 1.319 pessoas com diabetes tipo 2, no Nordeste do Brasil, 
em 2012, revelou que homens e mulheres realizavam o teste de glicemia capilar quase que na mesma proporção e quando questionados quanto ao conhecimento da importância da prática, apenas pouco mais da metade tiveram respostas afirmativas (SOUSA, et al., 2015). No México, pesquisadores observaram que em todos os aspectos avaliados no estilo de vida, entre homens e mulheres, os homens se mantiveram abaixo da média. O nível de controle glicêmico está englobado na categoria conhecimento e este foi classificado como regular entre ambos os sexos (Sánchez, Lira, Perales, \& Alonso, 2018).

\section{Comportamento alimentar versus o autocuidado}

Tanto homens quanto mulheres relataram uma alimentação diária inadequada ao reconhecer suas falhas, como a ingestão de alimentos que prejudicam sua saúde. A ingestão de alimentos em grande quantidade foi justificada pelos sintomas de ansiedade. Ainda, a rotina habitual de alimentação é rompida quando estão juntos da família.

[...] Café da tarde é a mesma coisa da manhã: café preto, outro pãozinho. Eu sou muito do pão. É aí que está o erro também. Às vezes eu como dois, três pãezinhos no café da manhã e da tarde também. (M14)

[...] À tarde eu abuso um pouquinho, como um pão inteiro [...] que eu não deveria por causa do diabetes. Faz mal. Sei que faz mal esse pão para o açúcar. Mesmo sabendo, faço isso, mas não deveria fazer. E a noite, ao invés de jantar, tomo café com um pãozinho também. (F4)

Como muito. Até sem a família. Agora que estou tomando um remédio para ansiedade. Pedi para o médico porque eu como muito mesmo. (F6)

[...] Às vezes só quando tem churrasco [em família], alguma coisa assim, aí modifica [risos]. (M11)

De casa? [Família] Daí eu espalho um pouquinho, esbanjo [risos]. (F9)

Por outro lado, identificou-se a manutenção da alimentação mais adequada quando a refeição é realizada junto dos familiares. Verificou-se que a família pode dar um apoio no que se refere à questão alimentar.

[...] Até faço mais coisas quando estou com eles todos, porque minhas filhas precisam de uma alimentação mais balanceada, aí dou uma caprichada. (F3)

Todo mundo lá se habituou a comer tudo igual a mim. Até arroz integral. Lá em casa todo mundo é na dieta. (M5)

Quanto às restrições alimentares para DM2, foram mencionados apenas os carboidratos como doce, açúcar, pão, massa e batata. E dessas restrições, quase nenhuma era praticada na vida cotidiana.

Que eu saiba que não pode comer pão branco, massa, arroz também, arroz branco. E doce, refrigerante, mais ou menos isso. (M11)

Bolo, doce, bala, essas coisas assim que a gente não pode comer. Massa, arroz demais também não, batata... (F2)

[...] Refrigerante eu não tomo. Doce, de vez em quando. (F1)

Procuro comer menos pão branco. (M3) 
O DM2 é uma síndrome que pode ser evitada e controlada a partir de mudanças no estilo de vida, como por exemplo, relacionadas à alimentação. $\mathrm{O}$ carboidrato foi a primeira restrição a ser lembrada pelos participantes, poucos referiram fritura, que aumenta o risco para doenças cardiovasculares, ou o sal que favorece a hipertensão. Ambas comorbidades associadas ao diabetes podem aumentar os índices de mortalidade (Sociedade Brasileira de Diabetes, 2017). Um estudo realizado na Europa constatou que o aumento da ingesta de alguns grupos alimentares, de forma controlada, como grãos integrais, vegetais, frutas e laticínios, podem modificar significativamente o risco de DM2, ainda mais se associada a uma redução de carnes vermelhas e processadas, bebidas açucaradas e ovos (Schwingshackl, et al., 2017).

Uma pesquisa realizada na Espanha, em 2016, com homens e mulheres que tinham DM2, identificou que os participantes acreditavam não terem informações e recursos para suprir as necessidades nutricionais. Ainda, foi constatado a ingestão excessiva de doces e gorduras saturadas, o que não coincide com a dieta mediterrânea prevista para a localidade. A pouca aceitação da dieta pode ser justificada pela falta de comunicação entre profissionais, pacientes e familiares (Lillo, \& Rodríguez, 2018).

Por outro lado, um estudo realizado no Distrito Federal em 2018, identificou que a maioria dos participantes não tiveram dificuldades em mudar o estilo de vida referente aos hábitos alimentares. Esse grupo de participantes fizeram ajustes na dieta, como a redução de doces, álcool e a quantidade dos alimentos ingeridos. Ainda que seguissem pouco o plano alimentar prescrito por profissionais de saúde, demonstraram comprometimento satisfatório com o autocuidado, promovendo uma boa manutenção da saúde (Silva, \& Alves, 2018).

\section{A rotina de vida versus a realização de atividade física}

Homens e mulheres referiram trabalhar tanto fora quanto em casa, alguns somente no lar. Identificou-se no depoimento dos participantes que a rotina de vida interferiu na prática de atividades físicas, de forma que as mulheres, por estarem mais envolvidas com as atividades domésticas, deixaram de fazer exercícios físicos, enquanto que os homens relataram maior assiduidade nessa prática.

Eu trabalho, trabalho bastante. Me exercito bastante no trabalho. Mas fora isso, exercícios físicos não. (F3)

Eu sou dona de casa e cuido do meu marido. Cuido dos medicamentos dele, os meus que a gente tem que tomar, as consultas, os exames que a gente tem que fazer e é isso. Meu dia a dia é isso. Cuido mais dele do que de mim. (F2)

Cuido da casa e cuido do men esposo que teve um AVC. Ele fez uma cirurgia, teve um aneurisma depois teve um AVC, aî não caminha, não fala, tenho que cuidar dele. Cuido dele, faço tudo. (F7)

Praticamente nada. Só em casa. É eu e minha sobrinha. A casa que eu arrumo, faço almoço, essas coisas, rotina de casa. $($ M11)

Eu faço peso, faço abdominal, ponte, várias coisas, caminho. (M12)

O estilo e a rotina de vida podem interferir diretamente na realização ou não de atividades físicas e isso impacta diretamente na saúde das pessoas com doenças crônicas, nesse caso o DM2. A prática de atividade física em conjunto com mudanças de hábitos cotidianos e adesão ao tratamento são fatores indispensáveis para o controle do diabetes e suas comorbidades, essas atitudes devem ser estimuladas pelos profissionais de saúde (Kolchraiber, et al., 2018).

Um estudo realizado em Brasília, entre 2001 e 2015, comprovou que a atividade física refletiu em resultados positivos no tratamento do DM2, influenciando de forma eficaz a diminuição dos níveis de glicemia capilar e de hemoglobina glicada, se 
equiparado aos resultados anteriores dos participantes da pesquisa. Contudo, para que se obtenha vantagens com a atividade física no tratamento da patologia, é necessária uma regularidade em sua prática e que seja acompanhado por um profissional habilitado, tendo em vista as alterações na glicemia que podem ocorrer durante o exercício (Soares, 2016).

Destaca-se o depoimento de uma mulher, que justifica a não realização de atividade física porque seu companheiro a proíbe com medo que ela sofra uma queda.

Olha, deveria, mas é que esse senhor que estou, ele não sai a caminhar comigo e ele não deixa eu sair sozinha. Não deixa com medo que eu vá cair, que é isso e aquilo, que pode me dar tontura, porque tem vezes que me dá umas tonturas e eu caio... (F4)

O risco de quedas e o medo gerado por ele podem gerar um sentimento de privação entre as pessoas que convivem com o DM2. Um estudo realizado em São Paulo entre 2010 e 2013, utilizando um grupo controle com DM2 e outro de pessoas que não tinham a referida patologia, verificou que pessoas com diabetes têm mais episódios de tontura relacionados a aspectos físicos, emocionais e funcionais do que as pessoas que não convivem com a doença. O medo de cair foi maior entre os pacientes com diabetes do que naqueles que não a possuem. Ainda, o fator mais prevalente de quedas foi o desequilíbrio corporal provocado por episódios de tontura crônica, que possivelmente pode estar relacionado com a neuropatia diabética (SOUZA, et al., 2018).

Por outro lado, é preciso atentar para as possíveis situações de proibição que podem estar associadas às desigualdades de gênero. Ao longo da história, o cenário machista sempre esteve tentando controlar o comportamento das mulheres. É possível observar tais atitudes dos homens quando eles tentam determinar os locais onde as mulheres podem frequentar, as roupas que são apropriadas para uma mulher recatada e as posições sociais que elas podem ocupar (Mello, 2018). Sendo assim, essas influências socioculturais também podem interferir no manejo do DM2.

\section{Considerações Finais}

Ao conhecer o autocuidado de homens e mulheres com Diabetes Mellitus tipo 2 foi possível identificar que apesar dos participantes apresentarem comportamentos de autocuidado similares, no que diz respeito a realização de atividade física se evidenciou uma maior adesão entre os homens. Esse fato, diferencia-se da expectativa socialmente imposta de que as mulheres se cuidam mais que os homens. A razão para esse distanciamento das mulheres na realização de exercícios físicos pode ser explicada pelo maior envolvimento das mulheres com as tarefas do lar e cuidados consigo e com os outros.

Além disso, identificou-se que a percepção dos participantes sobre seu autocuidado é incondizente com as reais práticas cotidianas, pois eles referiram realizar um bom autocuidado, mas relataram ingerir alimentos inadequados e realizar pouca atividade física. Ainda assim, o autocuidado ficou mais centrado na redução da ingesta de carboidratos, na realização de exercícios físicos, no uso correto das medicações, no controle de glicose e no cuidado com os pés. Também, reconheceram as dificuldades enfrentadas em relação ao seu autocuidado, admitindo que poderiam melhorar.

Assim, para melhorar o autocuidado das pessoas com diabetes, faz-se necessário um maior engajamento e conscientização dos mesmos sobre sua saúde para se atingir as mudanças necessárias no estilo de vida. Esse processo pode ser facilitado por meio de intervenções dos profissionais da saúde, em especial, da enfermagem, no que diz respeito à educação em saúde dos indivíduos, levando em consideração as questões de gênero existentes que podem influenciar o autocuidado, tendo em vista que homens e mulheres procuram os sistemas de saúde de maneiras diferentes. Também a criação de grupos de apoio, com olhar centrado na individualidade da pessoa com DM2 e em seu contexto sociocultural e econômico, podem favorecer a educação com relação a patologia. 
Ressalta-se que o estudo não se esgota neste trabalho, de modo que sugere-se a futuras pesquisas a ampliação da amostra com mais parâmetros de avaliação, como aspectos cognitivos a serem investigados, de modo a trazer melhorias na assistência da população em questão. Além disso, o atendimento ofertado pelos serviços de saúde sofreu alterações em virtude do cenário atual de pandemia da COVID-19, o que gera consequências em diversos segmentos da sociedade, em especial às pessoas que convivem com doenças crônicas, as quais necessitam de um olhar ainda mais integral e humanizado em um período em que muitas das atividades assistenciais estão temporariamente suspensas. Assim, torna-se pertinente a realização de estudos futuros acerca dessa temática durante esse período excepcional.

\section{Referências}

Araújo, E. S. S., et al. (2018). Cuidado de enfermagem ao paciente com diabetes fundamentado na Teoria de King. Revista Brasileira de Enfermagem, 71 (3).

Bardin, L. (2011). Análise de conteúdo. Tradução de Luís Antero Retos, Augusto Pinheiro. Edições 70.

Brasil. (2013). Ministério da Saúde. Estratégias para o cuidado da pessoa com doença crônica: diabetes mellitus. Brasília: Ministério da Saúde. https://bvsms.saude.gov.br/bvs/publicacoes/estrategias_cuidado_pessoa_doenca_cronica_cab35.pdf.

Brasil. (2018). Ministério da Saúde. Sobre a Vigilância de DCNT. http://www.saude.gov.br/noticias/43036-sobre-a-vigilancia-de-dcnt.

Costa, L. J. S. L., et al. (2016). Autocuidado dos adultos jovens com diabetes mellitus tipo 2. Revista Enfermagem UFPE on line, 10 (11), $3875-3882$. Recuperado de: https://periodicos.ufpe.br/revistas/revistaenfermagem/article/viewFile/11468/13307.

Cruz, R. R., et al. (2019). Avaliação de comorbidades e uso de medicamentos em pacientes com Diabetes Tipo 2 (DM2). Revista de Iniciação Científica e Extensão, 2 (1), 14. https://redib.org/Record/oai_articulo2078140-avalia\%C3\%A7\%C3\%A3o-de-cormobidades-e-uso-de-medicamentos-em-pacientes-comdiabetes-tipo2-dm2

Fernandes, S. S. C., Damascena, R. S., \& Portela, F. S. (2019). Avaliação da adesão ao tratamento farmacológico de idosos portadores de Diabetes Mellitus Tipo II acompanhados em uma Rede de farmácias de Vitória da Conquista-Bahia. Revista Multidisciplinar e de Psicologia, 13 (43), 241-263. https://idonline.emnuvens.com.br/id/article/view/1522.

Hoerpers, N. J., Roldão, G. S., Fernandes, P. R., Dimer, L. M., \& Pavei, S. R. P. (2019) Autocuidado das pessoas com diabetes mellitus tipo II em Estratégia Saúde da Família. Inova Saúde, 8 (2), 116-137. http://periodicos.unesc.net/Inovasaude/article/view/3458.

International Diabetes Federation. IDF diabetes atlas. (8th ed.), Brussels: International Diabetes Federation, 2017.

Kolchraiber, F. C., et al. (2018). Nível de atividade física em pessoas com diabetes mellitus tipo 2. Revista Cuidarte, 9 (2), $2105-2116$. https://pesquisa.bvsalud.org/portal/resource/pt/biblio-963430.

Lillo, M., \& Rodriguez, P. (2018). Valoración de la adherencia del paciente diabético al consejo nutricional y evaluación de mejoras tras su reeducación: una investigación-acción en la costa mediterránea. Revista chilena de nutrición, 45 (3), 205-215. http://rua.ua.es/dspace/handle/10045/79327.

Lisley, T., et al. (2019). Aspectos epidemiológicos do diabetes mellitus em Sergipe. Congresso Internacional de Enfermagem, 1 (1). https://eventos.set.edu.br/cie/article/view/11828.

Martins, M. M. F., \& Rodrigues, M. L. (2019). Diabetes: Adesão ao tratamento e o papel da família a essa nova realidade. Revista de Atenção à Saúde, 17 (59).

Mello, P. B. (2018). A repetição da violência doméstica contra a mulher: aspectos históricos, sociais e psicológicos. Trabalho de Conclusão de Curso (Psicologia) - Universidade Regional do Noroeste do Estado do Rio Grande do Sul, 2018. https://bibliodigital.unijui.edu.br:8443/xmlui/handle/123456789/4846.

Moura, E. C., Gomes, R., \& Pereira, G. M. C. (2017). Percepções sobre a saúde dos homens numa perspectiva relacional de gênero, Brasil, 2014. Ciência \& Saúde Coletiva, 22 (1), 291-300. http://www.cienciaesaudecoletiva.com.br/artigos/percepcoes-sobre-a-saude-dos-homens-numa-perspectiva-relacional-degenero-brasil-2014/15358?id=15358.

Oka, M., Laurenti, C. (2018). Entre sexo e gênero: um estudo bibliográfico-exploratório das ciências da saúde. Saúde e Sociedade, 27, 238-251. https://pesquisa.bvsalud.org/portal/resource/pt/biblio-962565.

Pimentel, T. S., \& Marques, D. R. S. (2019). Atuação do enfermeiro no controle da neuropatia periférica em pacientes portadores de diabetes mellitus tipo 2. Ciências Biológicas e da Saúde, 5 (2), 213. https://periodicos.set.edu.br/cadernobiologicas/article/view/6626.

Polit, D. F., \& Beck, C. T. (2011). Fundamentos de pesquisa em enfermagem: avaliação de evidências para a prática da enfermagem. Artmed Editora.

Ribeiro, V. S., \& Nunes, M. J. C. (2018). Pé Diabético: Conhecimento e Adesão às Medidas Preventivas. Rev. Cient. Esc. Estadual Saúde Pública Goiás “Cândido Santiago”, 4 (2), 156-169. http://www.revista.esap.go.gov.br/index.php/resap/article/view/95.

Sánchez, R. R., Lira, A. J., Perales, M. S., \& Alonso, L. R. M. (2018). Nivel de conocimientos, estilos de vida y control glucémico en pacientes con diabetes mellitus tipo 2. Revista ENE de Enfermería, 12 (1). http://www.ene-enfermeria.org/ojs/index.php/ENE/article/view/757. 
Research, Society and Development, v. 10, n. 1, e27210111675, 2021 (CC BY 4.0) | ISSN 2525-3409 | DOI: http://dx.doi.org/10.33448/rsd-v10i1.11675

Schwingshackl, L. et al. (2017). Food groups and risk of type 2 diabetes mellitus: a systematic review and meta-analysis of prospective studies. European Journal Epidemiology, 32 (5), 363-375. https://pubmed.ncbi.nlm.nih.gov/28397016/.

Silva, S. A., \& Alves, S. H. S. (2018). Conhecimento do diabetes tipo 2 e relação com o comportamento de adesão ao tratamento. Estudos Interdisciplinares em Psicologia, 9 (2), 39-57. http://pepsic.bvsalud.org/scielo.php?script=sci_arttext\&pid=S2236-64072018000200004\&lng=es\&nrm=iso.

Silva, W. I. dos S., et al. (2020). Conhecimento de pessoas diabéticas como fator preditivo para a adesão do autocuidado e controle glicêmico. Research, Society and Development, 9 (10). http://dx.doi.org/10.33448/rsd-v9i10.8474

Soares, R. D. J. (2016). A influência da atividade física nos níveis glicêmicos de idosos diabéticos tipo 2.23 f. Trabalho de Conclusão de Curso (Bacharelado em Enfermagem) - Universidade de Brasília, Brasília, 2016. https://bdm.unb.br/handle/10483/19471.

Sociedade Brasileira de Diabetes. Diretrizes da Sociedade Brasileira de Diabetes 2017-2018. Organização: José Egídio Paulo de Oliveira, Renan Magalhães Montenegro Junior, Sérgio Vencio. Editora Clannad, 2017.

Sousa, J. T., et al. (2015). Autocuidado e parâmetros clínicos em pacientes com diabetes mellitus tipo 2. Revista Rene, 16 (4), 479-485. http://www.repositorio.ufc.br/bitstream/riufc/14408/1/2015_art_jtsousa.pdf.

Souza, A. C., et al. (2018). Equilíbrio postural e acidentes por quedas em diabéticos e não diabéticos. Revista Brasileira de Saúde Funcional, 5 (2), 30. http://www.seer-adventista.com.br/ojs3/index.php/RBSF/article/view/973.

Teston, E. F., Sales, C. A., \& Marcon, S. S. (2017). Perspectivas de indivíduos com diabetes sobre autocuidado: contribuições para assistência. Escola Anna Nery Revista de Enfermagem, 21 (2), 1-8. https://www.semanticscholar.org/paper/Perspectivas-de-indiv\%C3\%ADduos-com-diabetes-sobre-para-TestonSales/7c0304df40132ae753a8e60b3bea6720e8e8f5a3.

Thiry-Cherques, H. R. (2009). Saturação em pesquisa qualitativa: estimativa empírica de dimensionamento. Revista PMKT, 3 (2), $20-27$. http://www.revistapmkt.com.br/Portals/9/Edicoes/Revista_PMKT_003_02.pdf.

Trajano, S. S., et al. (2018). Percepção de pacientes com diabetes sobre o autocuidado. Revista Brasileira em Promoção da Saúde, 31 (3). https://periodicos.unifor.br/RBPS/article/view/7598.

World Health Organization. Global status report on noncommunicable diseases 2014. Geneva: World Health Organization, 2014 